\title{
OGD2011 - Requirements Analysis for an Open Data Strategy (in Austria)
}

\author{
Martin Kaltenböck \\ Semantic Web Company, OGD Austria, Lerchenfelder Gürtel 43, 1160 Vienna, Austria \\ m.kaltenboeck@semantic-web.at
}

\begin{abstract}
The OGD2011 project launches the 1st Open Government Data Conference Austria and brings together international and national experts in the area of Open Government Data as well as representatives of the main four open data stakeholder groups: politicians, citizens, industry and the public administration in Austria for the first time in 2011 in Vienna. But OGD2011 is more than a conference: in the course of four workshops with representatives of the 4 mentioned stakeholder groups in Spring 2011 the expectations \& requirements of these groups as well as challenges \& threats and open questions \& important issues of the open data process in Austria have been evaluated and analysed. As an output of this analysis as well as of the conference the OGD2011 project team publishes the OGD White Book Austria. This paper gives an overview over the outcomes of the OGD2011 project.
\end{abstract}

Keywords: open government data, open data, requirements, analysis.

\section{Introduction}

To analyse the requirements of an open government data strategy for Austria as well as to evaluate expectations, challenges and threats of open data itself, relevant representatives of the four main stakeholder groups were identified and invited to stakeholder workshops: politicians, citizens, industry and public administration (as well as the additional stakeholder groups: media and academia). The participants for these workshops were chosen to ensure a homogeneous workshop group per stakeholder workshop (e.g. for the politicians workshop members of all Austrian main parties were invited as well as representatives from local, regional and national governments). Every workshop was moderated by two persons of the area of public administration research and was documented by 2 members of the OGD2011 team. In the forefront of the workshops the participants have been invited to prioritize the relevant topics of open data for their respective workshop (12 topics were given for this preparation). On the basis of this prioritization the workshop agendas have been created and the workshops have been accomplished along the highest weighted open data topics of the prioritization of the respective stakeholder group. The minutes of the 4 workshops have been compacted and reports have been created out of the minutes. As a final step these reports have been compared with each other as well as populated by additional research results, additional publications and the outcome of about $\sim 40$ meetings with representatives of the 4 stakeholder groups that took place along the 
OGD2011 process. The outcome of this is published in the OGD2011 White Book Austria. As follows you can find the main identified topics to keep in mind for an open data process/strategy.

\section{Need for Definitions}

As one of the first steps for an open data strategy / process it was identified that there is a strong need to create definitions to ensure further discussion on open data on the basis of a common understanding. As there are different legal issues and frameworks as well as cultures and languages in the EU 27 countries these definitions need to be created for every nation. The following terms needed a deeper look in the OGD2011 project: open government, open government data, linked open government data, public sector information. After the clarification of such definitions together with the relevant stakeholders it is on the one hand possible to trigger a political process on open data as well as on the other hand to ensure that all stakeholders as well as the community and the interested public are talking about the same things.

\section{Open Government: Transparency and Democracy, Participation and Collaboration}

When starting to discuss open data as a measure for the digital infrastructure of a country the stakeholders that have to become active first are politicians as well as the public administration. One of the strongest arguments of open data is to enable open government: more transparency of processes and decisions made by the politics and the administration, what then becomes a key factor for better democracy on the basis of a better understanding between citizens - politicians - public administration. This new open government paradigm could also lower disenchantment with politics and can be achieved by a stronger involvement (participation) of the citizens in public processes and decision making processes as well as in enabling collaboration between all open data stakeholders. To ensure this open government there is a strong need for a commitment on open data by the highest levels of politics as well as public administration.

\section{Legal Issues}

A further criteria that is very important in the course of an open data process for a country is to identify and discuss legal issues for this open data strategy. As laws and regulations differ very strong between EU27 countries these legal issues can hardly be transferred from one country to the other. Existing laws and regulations have to be evaluated and maybe adapted for this. In many EU27 countries the ground principles of the EPSI directive (European Public Sector Information Directive, came into force on 17 November 2003 approved by the Council of Ministers - see: http://ec.europa.eu/information_society/policy/psi/index_en.htm) have already been implemented into national laws. That means that these laws could be a very good 
starting point for additional needed legal changes in information re-use. For example in Austria the 'IWG: Informationsweiterverwendungsgesetz' (the IWG came into force on 18 November 2005, see: http://www.ris.bka.gv.at/) is the existing law for this. Furthermore the issue of pro-active information provision by public administration as well as public liability and disclaimers of liability for open data need to be discussed and regulated in the course of a nationwide open data strategy.

\section{Impact on Society}

As open data is a relatively novel field of data and information provision there is also a tremendous impact on society. New cultural techniques are needed in several fields: in the field of data interpretation, analysis and visualization - along the whole data handling mechanisms and techniques. Because open data is not useful if the data is not used! Furthermore the handling of crowd sourcing mechanisms, of stronger ways of e-participation and collaboration between citizens, politics and public administration needs to be taken into account and last but not least there is also a need for a change inside the public administration - as open data changes the used ways of data management and publishing as well as it the lowers the borders between citizens and the public administration. That means on the one hand that we do need to discuss and implement new methods in media and data literacy (from ground school up to adult education) as well as on the other hands we need to discuss and manage change processes inside of the public administration. Furthermore issues of privacy and data protection need to be discussed and solved here.

\section{Innovation and Knowledge Society}

One main issue why we are talking about Open Government Data is to strengthen the information society in Europe by enabling innovation and the knowledge society. This means that it is of very high importance to ensure that there is free available data to look at, take it and play with it to ensure these innovation mechanisms. As Prof. Nigel Shadbolt of the University of Southampton, one of the leading experts in UK in the open data process, pointed out: 'open the data and the Apps will flow'.

Important to mention here is to think also about technical issues of Open Government Data: putting the term 'Linked' in front of it supports strongly the creation of knowledge out of interlinked data. Linked Open (Government) Data principles ensure that information (and things) is interlinked and thereby context is provided and can be used to create new knowledge.

\section{Impact on Economy/Industry}

When basic structures of an Open Government Data strategy are discussed for a country it is very important not to forget the stakeholder group of industry to be involved in the Open Data strategy and the OGD process as well as in the concrete implementation/realization. Industry will benefit of Open Government Data - as information is one of the most important goods/resources of our service-oriented economy in Europe 
(as it is also for industry for e.g. enabling better decision making in the area of market intelligence et al.). The impact of Open Government Data on economy is split into direct impact (as commercial revenues and thereby taxes, foundation of new companies, etc.) and indirect economic impacts (as lower unemployment and several areas of the national economy as e.g. health and many others). This area of economic impact needs to be studied more in detail as it lacks a little bit of figures and measurements at the moment - but this can be done in the course of the 1st year of an open data strategy. As a first point it is very important to involve the economy/industry of a country as an important stakeholder.

\section{Licenses, Models for Exploitation, Terms of Use}

Coming from economic impact initiated by Open Government Data there is a strong need to discuss all issues of licensing as well as exploitation models and terms of use for this Open Government Data. Providing licenses ensures several issues: 1) giving the users of the data legal compliance as it is clear what the users are allowed to do with the data, 2) giving the data provider a disclaimer of liability and 3) learn about the usage of the data (e.g. when a license includes the obligation to refer to the data source when using a data set). Based on this, exploitation models need to be discussed and developed: this is important on the one hand for the public administration as there are lots of data sets require a fee at the moment and on the other hand for the industry that wants to set up new business models on top of the data. Issues as 'freemium models' (some data for free to enable innovation and 'better data' cost a fee), service level agreements (permanence, performance, stability, timeliness, etc.) et al need to be discussed here.

\section{Data relevant Aspects}

Coming to the data itself then, there are lots of things to take into account. Type and scale of data that can be provided as Open Data - as a first step there is a strong need to manage an internal inventory of data in the public sector - said this does not mean that existing data sets cannot be provided as soon as possible at a single point of access (as for instance a data catalogue) to ensure to use the 'low hanging fruits' - but in the long run every data set needs a look at to decide whether to open it or not and when to open it and how to open it. The OGD2011 project shows, as well as a deeper look on existing open data activities, that to open up the 'basic data' is a very good approach (geo data, statistical data etc.) at the beginning to ensure the provision of a basic data infrastructure that users can work with.

Furthermore the following aspects need to be taken into account: formats, permanence \& timeliness, raw data, meta data, data granularity, completeness, data quality et al.

\section{Data Governance}

Beside the concrete aspects of the data itself there is also a lot to think about the data surroundings, the data governance. Origin and source of data, information on the 
process of data harvesting/collection, information about how to interpret (maybe provision of a reference interpretation) need to be given together with the data (best as a part of the provided meta data). For this topic the expertise of the respective domain experts is needed and highly recommended. Data governance is one of the most important topics to ensure an efficient usage of the data. For example an Open Government Data Stakeholder Survey of 2010/2011 managed by the EU project LOD2 (http://www.lod2.eu) has shown that the main issue of trusting a data set is the data sets origin / source.

\section{Applications and Use Cases}

Clearly the usage of the data is an important issue for every Open Data activity, too - because the data is absolutely useless if the data is not used. Taking a look at best practices in EU27, the US, Australia and New Zealand can help a lot here. Beside the above mentioned issues of data literacy there is a strong need to pro-actively involve users in the Open Data process. Competitions can help a lot to generate visibility and to ensure a first output in the area of use cases \& ideas, data analysis and visualizations and new and innovative applications on the open(ed) data. The best applications to name here are apps that generate a benefit for both: the citizens as well as the public administration.

\section{Technological Aspects}

Last but not least we do need a deeper look into the area of infrastructure and technology to really enable the power of Open Government Data. As mentioned before it needs to be mentioned again: Open (Government) Data is a measure for the digital infrastructure of a country - as the building of roads or as electricity - as we are talking about our information and knowledge society in Europe! On top of this infrastructure data integration, services and application et al. can be developed and realized. To ensure to bring the potential of Open Data to its full blown power it is important to keep in mind a few things to A) ensure the widest range of usage as well as B) to ensure the most useful \& powerful way of usage. Said this means to A) provide data in several formats and B) also provide an API is important to ensure that as many users as possible are able to use the data, C) the use of open formats and standards for Open Data is important to lower barriers ( $\&$ costs) for the usage, D) the provision of a single point of access to the data (a data portal / catalogue) where users can find all of the data very quick and easy, E) provision of all data in human AND machine readable formats, F) in the mid- and long-term provision of the data following the $\mathrm{W} 3 \mathrm{C}$ linked open data principles (see: http://en.wikipedia.org/wiki/Linked_Data) to enable the most powerful way for the usage of data (including the provision of context) as well as to ensure interoperability. Linked Data is also the direction the European Commission goes: so that interoperability between EU27 can be enabled in the future using linked open data standards and principles. This approach can also be seen by Sir Tim Berners Lee the director of W3C in his 5 star model of Open Government Data (http://www.w3.org/DesignIssues/LinkedData.html). 


\section{Conclusion}

Summarising the output of the OGD2011 project there are several issues and areas that need to be taken into account when starting an Open Government Data strategy for a nation (or city or region etc). This starts at the need of definitions over open government and legal issues to issues around the impact on society and economy/industry. Beside this data relevant issues need to be analysed, discussed and clarified as licenses, data governance and technological aspects. From a planning perspective a 2 phase approach seems to be the right one:

a) starting with low hanging fruits by providing existing open data at a single point of access (some basic data sets as postal codes, geospatial data, statistical data etc.) as early as possible and

b) for mid-term and long-term approach to start a comprehensive project by taking all above mentioned issues into account and see Open Government Data asa measure for the digital infrastructure of a country. 